\title{
Efficient Atomic Clocks Operated with Several Atomic Ensembles
}

\author{
J. Borregaard* and A. S. Sørensen ${ }^{\dagger}$ \\ QUANTOP, The Niels Bohr Institute, University of Copenhagen, Blegdamsvej 17, DK-2100 Copenhagen Ø, Denmark
} (Received 24 April 2013; revised manuscript received 12 July 2013; published 27 August 2013)

\begin{abstract}
Atomic clocks are typically operated by locking a local oscillator (LO) to a single atomic ensemble. In this Letter, we propose a scheme where the LO is locked to several atomic ensembles instead of one. This results in an exponential improvement compared to the conventional method and provides a stability of the clock scaling as $(\alpha N)^{-m / 2}$ with $N$ being the number of atoms in each of the $m$ ensembles and $\alpha$ a constant depending on the protocol being used to lock the LO.
\end{abstract}

DOI: 10.1103/PhysRevLett.111.090802

PACS numbers: 06.30.Ft, 03.65.Ud, 03.65.Yz, 06.20.Dk

Atomic clocks provide very precise time measurements useful for a broad range of areas in physics. The quantum noise of the atoms limits the stability of atomic clocks, resulting in the standard quantum limit where the stability scales as $1 / \sqrt{N}$, with $N$ being the number of atoms [1,2]. Various ways of improving the resolution have been suggested, such as using entangled states with reduced atomic noise [3-6] to push the resolution to the Heisenberg limit where it scales as $1 / N$ [7-12]. Another approach to increasing the stability is to use optical atomic clocks where the higher operating frequency leads to an improved stability [13-17]. Since an atomic clock is typically operated through Ramsey spectroscopy [18], the resolution can also be enhanced by increasing the Ramsey time $T$, resulting in an improvement scaling as $1 / \sqrt{T}$ [19-21]. For clocks with trapped atoms, where there are no other limitations, $T$ becomes limited only by the decoherence in the system. In practice, this decoherence often originates from the frequency fluctuations of the local oscillator (LO) used to drive the atomic clock transition [20]. Hence, the stability can also be increased by simply devising methods to increase the Ramsey period by stabilizing the LO [22].

In this Letter, we suggest a scheme where the frequency of the LO is locked to the atomic transition using several ensembles of atoms. This procedure allows increasing the Ramsey period each time another ensemble is used. As a result, we find that the stability of the clock can increase exponentially with the number of ensembles. Figure 1(a) illustrates the idea behind the scheme. The feedback of the first ensemble locks the frequency of the LO, thus reducing the noise to the atomic noise. Having reduced the noise in the LO, the second ensemble can be operated with a longer Ramsey time. Through a second feedback, the noise of the LO can be further reduced, as shown in the simulation in Fig. 1(b) (details are given later). The procedure can be extended to any number of ensembles, and for uncorrelated atoms, the stability of the $\mathrm{LO}$ will scale as $\sqrt{\gamma}\left(\gamma T_{1} N\right)^{-m / 2}$, where $m$ is the number of ensembles (each containing $N$ atoms), $\gamma$ is a parameter characterizing the frequency fluctuations of the unlocked $\mathrm{LO}$, and $T_{1}$ is the Ramsey time of the first ensemble. Hence, the scheme can provide an exponential improvement in the stability with the total number of atoms. In order for the clock to be stable, we need $\gamma T_{1} \ll 1$, and hence the protocol requires a minimum number of atoms to improve the performance. With the conventional Ramsey protocol, we find that the scheme works for a minimum ensemble size of 20 atoms. To further optimize the performance of the scheme, we study an adaptive measurement protocol for estimating the LO frequency offset, which extends the applicability of the scheme down to ensembles with only 4 (7) atoms for white $(1 / f)$ noise in the LO. This makes the scheme relevant for atomic clocks based on trapped ions, which are typically constructed with only a few ions [19]. A related procedure involving multiple measurements on a single ensemble was proposed in Ref. [22]. By using multiple ensembles, our procedure avoids disturbances from the measurements
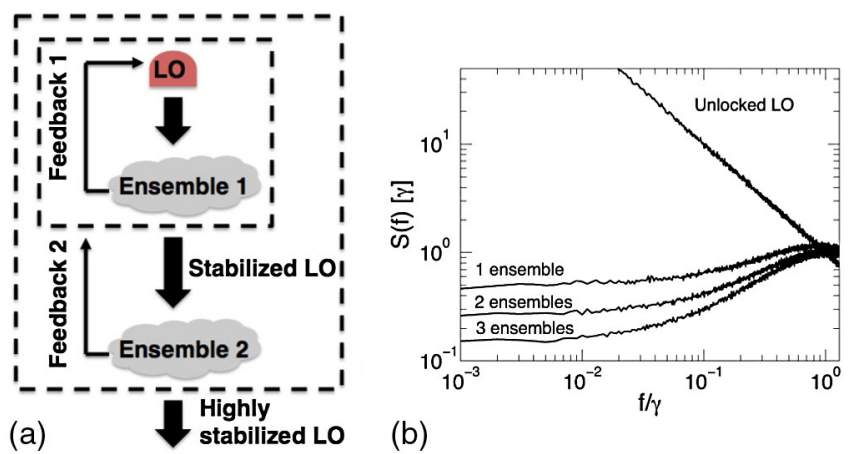

(b)

FIG. 1 (color online). (a) Illustration of locking the LO using several ensembles. The feedback of the first ensemble stabilizes the LO such that the second ensemble can be operated with a longer Ramsey time. The feedback from the second ensemble then further stabilizes the LO. (b) Numerical simulation of the noise spectrum $S(f)$ of the LO when locked to between 1 and 3 ensembles. The data were simulated as described in the text for $N=20$ and $T_{1}=0.1 / \gamma$ for the conventional Ramsey scheme. The first feedback lowers the noise of the LO and whitens the spectrum, even though the unlocked LO was assumed to be subject to $1 / f$ noise. The second and third feedbacks further lower the noise of LO by a constant factor. 
affecting later measurements. Recently and independently from this work, a manuscript appeared which treats essentially the same locking scheme that we suggest [23]. Taking the different figures of merit into account, that work arrives at results consistent with ours.

We will now describe the locking of the LO to the atomic transition using Ramsey spectroscopy. We model an ensemble of $N$ atoms as a collection of spin- $1 / 2$ particles with total angular momentum $\vec{J}$. We define the angular momentum operators $\hat{J}_{x}, \hat{J}_{y}$, and $\hat{J}_{z}$ in the usual way, and initially the atoms are pumped to have $\langle\vec{J}\rangle$ along the $z$ direction $\left\langle\hat{J}_{x}\right\rangle=\left\langle\hat{J}_{y}\right\rangle=0$. In Ramsey spectroscopy, the atoms are illuminated by a near-resonant $\pi / 2$ pulse from the LO, followed by the Ramsey time $T$ of free evolution, and finally another near-resonant $\pi / 2$ pulse is applied. The Heisenberg evolution of $\hat{J}_{z}$ is $\hat{J}_{3}=\cos (\delta \phi) \hat{J}_{y}+$ $\sin (\delta \phi) \hat{J}_{z}$, where $\delta \phi=\delta \omega T$ is the acquired phase of the LO relative to the atoms. At the end of the Ramsey sequence, $\hat{J}_{3}$ is measured and used to make an estimate $\delta \phi^{e}=-\arcsin \left(2 \hat{J}_{3} / N\right)$ of $\delta \phi$. The feedback loop then steers the frequency of the LO towards the atomic transition by applying a frequency correction of $\Delta \omega=$ $-\alpha \delta \phi^{e} / T$ to the LO, where $\alpha$ sets the strength of the feedback loop. The operation of an atomic clock thus consists of repeating a cycle of initialization, Ramseysequence, measurement and feedback. The total time of this clock cycle is denoted as $T_{c}$, and we assume that $T_{c} \sim T$; i.e., we assume a negligible Dick noise [24].

We now consider an atomic clock with two atomic ensembles operated with different Ramsey times and show how this can improve the stability of the clock. These considerations can then easily be extended to several ensembles. Note that we assume the intrinsic linewidth of the atoms to be negligible, such that the atomic linewidth is only limited by the Ramsey time. The first ensemble is operated with Ramsey time $T_{1}$, and we assume that the second ensemble is operated with Ramsey time $T_{2}=n T_{1}$, where $n$ is an integer. We can make two discrete time scales describing ensembles one and two, respectively. Ensemble one is measured at $t_{k}=k T_{1}$, and ensemble two is measured at $t_{s}=s T_{2}=s n T_{1}$. The frequency offset of the LO between time $t_{k-1}$ and $t_{k}$ is then

$$
\delta \omega(t)=\delta \omega_{0}(t)+\Delta \omega_{1}\left(t_{k-1}\right)+\Delta \omega_{2}\left(t_{s-1}\right),
$$

where $\delta \omega_{0}(t)$ is the frequency fluctuation of the unlocked LO, $\Delta \omega_{1}\left(t_{k-1}\right)$ is the sum of the frequency corrections applied up to time $t_{k-1}$ from the first ensemble, and $\Delta \omega_{2}\left(t_{s-1}\right)$ is the sum of the frequency corrections applied up to time $t_{s-1}$ from the second ensemble $\left(t_{s-1} \leq t_{k-1}\right)$. The feedback loops are described by the equations

$$
\begin{aligned}
& \Delta \omega_{1}\left(t_{k-1}\right)=\Delta \omega_{1}\left(t_{k-2}\right)-\alpha \delta \phi^{e_{1}}\left(t_{k-1}\right) / T_{1}, \\
& \Delta \omega_{2}\left(t_{s-1}\right)=\Delta \omega_{2}\left(t_{s-2}\right)-\alpha \delta \phi^{e_{2}}\left(t_{s-1}\right) / T_{2},
\end{aligned}
$$

where $\delta \phi^{e_{1}}\left(t_{k-1}\right)$ and $\delta \phi^{e_{2}}\left(t_{s-1}\right)$ are the estimated phases from the first and second ensembles at times $t_{k-1}$ and $t_{s-1}$, respectively. Using Eq. (1), we can write the phase of the LO relative to the atoms of the second ensemble at time $t_{s}$ as

$\delta \phi_{2}\left(t_{s}\right)=\int_{0}^{T_{2}} d t^{\prime} \delta \omega\left(t_{s}-t^{\prime}\right)=\Delta \phi_{s-1}+\delta \tilde{\phi}\left(t_{s}\right)$,

where $\Delta \phi_{s-1}=\int_{0}^{T_{2}} \Delta \omega_{2}\left(t_{s-1}\right) d t^{\prime}$ is the accumulated phase due to the feedback of the second ensemble and

$$
\begin{aligned}
\delta \tilde{\phi}\left(t_{s}\right) & =\int_{0}^{T_{2}} d t^{\prime} \delta \tilde{\omega}\left(t_{s}-t^{\prime}\right) \\
& =\int_{0}^{T_{2}} d t^{\prime} \delta \omega_{0}\left(t_{s}-t^{\prime}\right)+\Delta \omega_{1}\left(t_{s}-t^{\prime}\right)
\end{aligned}
$$

is the accumulated phase due to the frequency oscillations of the LO when locked by the feedback of the first ensemble. For now, we assume that $T_{2} \gg T_{1}$, such that the feedback of the first ensemble has stabilized the LO, but later we will relax this assumption. From Eqs. (3) and (4), we then derive the difference equation

$$
\delta \phi_{2}\left(t_{s}\right)-\delta \phi_{2}\left(t_{s-1}\right)=\delta \tilde{\phi}\left(t_{s}\right)-\delta \tilde{\phi}\left(t_{s-1}\right)-\alpha \delta \phi^{e_{2}}\left(t_{s-1}\right) .
$$

From this expression, we see that the evolution of the second phase $\delta \phi_{2}$ is essentially driven by the noise of the stabilized LO from the first step $\delta \tilde{\phi}$ but is stabilized by the second feedback loop described by $\alpha \delta \phi^{e_{2}}$.

To solve Eq. (6), we need to characterize the width of the noise of the stabilized LO from the first stage $\left\langle\delta \tilde{\phi}^{2}\right\rangle=$ $\int_{0}^{T_{2}} d t \int_{0}^{T_{2}} d t^{\prime}\left\langle\delta \tilde{\omega}(t) \delta \tilde{\omega}\left(t^{\prime}\right)\right\rangle$. From Eqs. (2) and (5), we can derive a difference equation for $\delta \tilde{\phi}\left(t_{k}\right)=\int_{0}^{T_{1}} \delta \tilde{\omega}\left(t_{k}-t^{\prime}\right) d t^{\prime}$, which is the acquired phase of the LO relative to the first ensemble between time $t_{k-1}$ and $t_{k}$ (we can neglect the feedback from the second ensemble since $T_{2} \gg T_{1}$ ):

$$
\delta \tilde{\phi}\left(t_{k}\right)-\delta \tilde{\phi}\left(t_{k-1}\right)=\delta \phi_{0}\left(t_{k}\right)-\delta \phi_{0}\left(t_{k-1}\right)-\alpha \delta \phi^{e_{1}}\left(t_{k-1}\right) .
$$

Here, $\delta \phi_{0}\left(t_{k}\right)=\int_{0}^{T_{1}} \delta \omega_{0}\left(t_{k}-t^{\prime}\right) d t^{\prime}$ is the phase of the unlocked LO. In comparison to Eq. (6), we see that the evolution of the phase $\delta \tilde{\phi}$ is driven by the noise of the unlocked LO but is stabilized by the first feedback loop described by $\alpha \delta \phi^{e_{1}}$. To solve this equation, we follow Ref. [25], where the locking of the LO to a single ensemble is described. First, we derive a differential equation from Eq. (7) in the limit $N \gg 1$, treating $\hat{J}_{x}, \hat{J}_{y}$, and $\hat{J}_{z}$ as Gaussian variables and considering for now a LO subject to white noise. Assuming that the atoms start out in a coherent spin state, we can solve this equation to obtain

$$
\left\langle\delta \tilde{\phi}^{2}\right\rangle=T_{2} / N T_{1}=\tilde{\gamma} T_{2},
$$

where we have defined the parameter $\tilde{\gamma}=1 / N T_{1}$, which characterizes the noise of the stabilized LO. This noise is 
effectively white for both white and $1 / f$ noise in the unlocked LO [Fig. 1(b) and Ref. [25]]. The second ensemble thus sees an effective white noise in the LO with $\tilde{\gamma}=1 /\left(T_{1} N\right)$.

We now return to Eq. (6). Writing $\delta \phi_{2}(t) \sim \delta \omega(t) T_{2}$, the stability of the clock after running for a time $\tau \gg T_{2}$ is

$$
\sigma_{\gamma}(\tau)=\frac{1}{\omega \tau T_{2}}\left(\int_{0}^{\tau} d t \int_{0}^{\tau} d t^{\prime}\left\langle\delta \phi_{2}(t) \delta \phi_{2}\left(t^{\prime}\right)\right\rangle\right)^{1 / 2},
$$

where $\omega$ is the frequency of the atomic transition. Following similar arguments as before, we can derive and solve a differential equation from Eq. (6) to obtain an expresion for $\left\langle\delta \phi_{2}(t) \delta \phi_{2}\left(t^{\prime}\right)\right\rangle$. Inserting this into Eq. (9) and taking the limit of $\tau \gg T_{2}$ results in

$$
\sigma_{\gamma}(\tau)=\frac{1}{\omega} \sqrt{\frac{1}{\tau N T_{2}}}
$$

Equation (10) describes how the stability improves with $T_{2}$ and $N$. The longest $T_{2}$ we can allow is determined by how well the LO is stabilized by the first ensemble as contained in $\tilde{\gamma}$, and we parametrize it by $T_{2, \max }=\beta_{2} / \tilde{\gamma}$. In a similar fashion, we assume that $T_{1, \max }=\beta_{1} / \gamma$ for the first ensemble. With these parametrizations, we can express the stability as

$$
\sigma_{\gamma}(\tau)=\frac{1}{\omega} \sqrt{\frac{\gamma}{\tau N^{2} \beta_{1} \beta_{2}}}=\frac{1}{\omega} \sqrt{\frac{\gamma \beta_{1} / \beta_{2}}{\tau\left(N \gamma T_{1, \max }\right)^{2}}} .
$$

With white noise in the unlocked LO, we can pick $\beta_{1}=$ $\beta_{2}$. As previously noted, the noise of the LO will also be approximately white with $\tilde{\gamma} \sim 1 / N T_{1}$ after locking it to the first ensemble also for other types of noise, e.g., $1 / f$ noise. In that case, it is desirable to have $\beta_{2} \neq \beta_{1}$, but we still expect $\beta_{1} / \beta_{2}$ to be of order unity. Equation (11) shows that by locking the LO to two ensembles of uncorrelated atoms, the stability can be significantly improved. If $N \gamma T_{1} \gg 1$, the stability obtained from Eq. (11) is much better than the single ensemble result in Eq. (10) (with $T_{2} \rightarrow T_{1}$ ). The arguments leading to Eq. (11) can be generalized in a straightforward way to show that if the LO is locked to $m$ ensembles each containing $N$ atoms, the stability of the clock is $\sigma_{\gamma}(\tau)=$ $\sqrt{\left(\beta_{1} / \beta\right)^{(m-1)} \gamma /\left(\omega^{2} \tau\right)}\left(N \gamma T_{1, \max }\right)^{-m / 2}$ (since the noise of the LO is white after locking it to the first ensemble, we use $\beta=\beta_{2}=\cdots=\beta_{m}$ ). By continuing the procedure, we thus improve the stability exponentially.

In our analytical calculations above, we have assumed $N \gg 1$. To investigate the performance for smaller $N$, we simulate an atomic clock locked to between 1 and 4 atomic ensembles, each with atom numbers from $N=20$ to $N=100$. From the simulations, we can generalize to the case where the LO is locked to $m$ ensembles. We simulate the full quantum evolution of the atomic state through the Ramsey sequences and subsequent measurements and implement the feedback on the LO similar to the description in Eq. (1) and above. The assumption of $T_{2} \gg T_{1}$ can be relaxed by applying a phase correction in the measurement [26]. The number of atoms required in each ensemble to increase the Ramsey time by a factor $a$ at each level is set by the white noise level of the stabilized LO. Using Eq. (8) and remembering that $\beta$ parametrizes the maximal Ramsey time for white noise, we have that $T_{2} / N T_{1}=$ $\tilde{\gamma} T_{2}=\beta$. Assuming $T_{2}=a T_{1}$, we find that $N \sim a / \beta$ atoms are required in each ensemble to increase the Ramsey time by a factor of $a$ at each level. The minimum number of atoms required for our protocol to work is thus obtained by setting $a=2$.

To determine $\beta$, we investigate the errors that limit the Ramsey time $T$ for a LO subject to white noise characterized by $\gamma$. For experiments or simulations running with a fixed Ramsey time, there is always a finite probability that phase jumps large enough to spoil the measurement strategy occur since Ramsey spectroscopy with projective measurements is only effective for phases $\leqslant \pi / 2$. In our simulations, we see these phase jumps as an abrupt breakdown as we increase $T$. Simulating a clock running for a time $\tau=10^{6} T$ with a single ensemble of $N=10^{5}$ atoms, we see the stability increase with $T$ until a maximum of $T_{\max } \sim 0.1 / \gamma$ is reached. Increasing $T$ beyond this point results in a rapid decrease in the stability. From this, we conclude that Ramsey spectroscopy with projective measurements only allows for $\beta \sim 0.1$, and thus $N_{\min }=20$. To determine $\beta_{1}$ for an LO subject to $1 / f$ noise, we do a similar simulation where the noise spectrum of the LO is $S(f)=\gamma^{2} / f$ ( $f$ is frequency). From this simulation, we find that $\beta_{1} \sim 0.1$ as for white noise. Note that this construction introduces a weak (logarithmic) dependence on the number of steps that we simulate [26].

We have simulated clocks with an unlocked LO subject to both white and $1 / f$ noise with the constraint $\beta=0.1$. In Fig. 2, the stability of the clocks is plotted against the ensemble size $N$. Figure 2 confirms that the scheme works
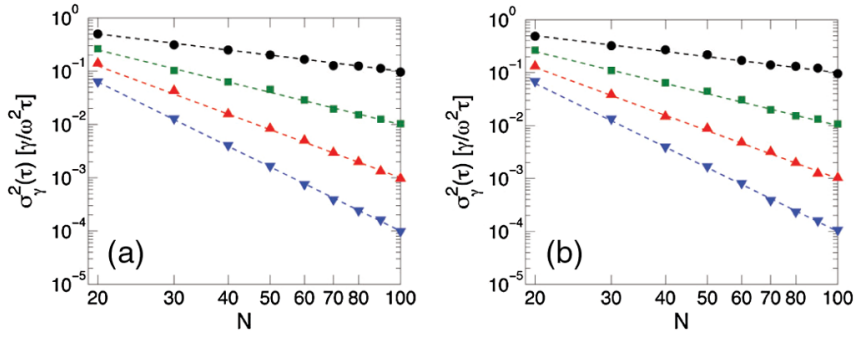

FIG. 2 (color online). The stability of atomic clocks for a LO subject to (a) white noise and (b) $1 / f$ noise. Circle, square, upward triangle, and downward triangle are the stability of a clock with the LO locked to $1,2,3$, and 4 ensembles containing $N$ atoms each. The clocks were simulated with $\beta_{1}=0.1$ and $T_{j}=n T_{j-1}$. Counting from the left (low $N$ ), the points are for integers $n$ from 2 to 10 . The dashed lines are the analytical calculations. 

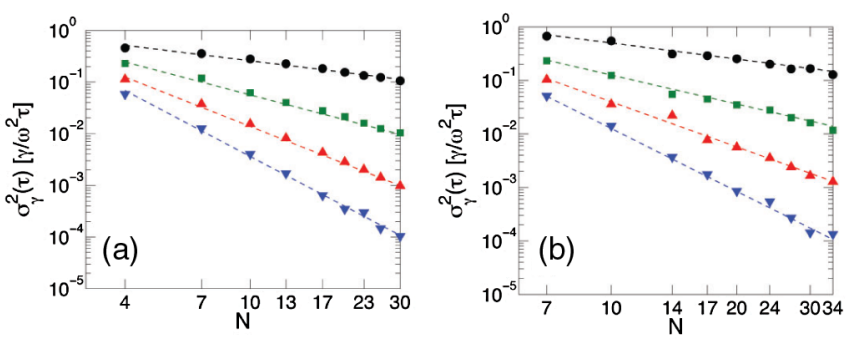

FIG. 3 (color online). The stability of atomic clocks with adaptive measurements for a LO subject to (a) white noise and (b) $1 / f$ noise. Circle, square, upward triangle, and downward triangle are the stability of a clock with the LO locked to 1,2, 3, and 4 ensembles of $N$ atoms each. The adaptive protocol allows for (a) $\beta_{1}=0.3$ and (b) $\beta_{1}=0.2$. The clocks were simulated with $T_{j}=n T_{j-1}$, and counting from the left (low $N$ ), the points are for integers $n$ from 2 to 10 . The dashed lines are fits of the simulated data.

down to atom numbers of $N=20$ where we gain a factor of $\sim 2^{m-1}$ in $\sigma_{\gamma}^{2}(\tau)$ by locking the LO to $m$ ensembles for both white and $1 / f$ noise. Furthermore, the numerical results are seen to agree nicely with the analytical calculations. We obtain practically the same long term stability for $1 / f$ noise as for white noise since the first feedback whitens the noise for small frequencies [cf. Fig. 1(b)].

The conventional Ramsey protocol considered so far has a lower limit of $N_{\min }=20$ in order for our protocol to work. This limit is due to the inability of the conventional protocol to effectively resolve phases larger than $\pi / 2$. In Ref. [27], we presented an adaptive protocol for estimating the phase, which effectively resolves phases $\lesssim \pi$. Again, simulating a clock running for a time $\tau=10^{6} T_{1}$ with a single ensemble of $N=10^{5}$ atoms, we find that this protocol enables us to extend the Ramsey time to $\beta \sim 0.3$ for white noise and to $\beta_{1} \sim 0.2$ for a LO subject to $1 / f$ noise [26]. However, the type of weak measurements described in Ref. [27] is hard to implement for ensembles of few atoms. We have therefore modified the protocol such that individual atoms are read out one at a time, and a Bayesian procedure similar to that of Refs. [28,29] is used for the phase estimation and atomic feedback. We perform intermediate feedbacks during the measurements to rotate the atomic state to be almost in phase with the LO. Because of the rotations, the protocol can resolve phases $\lesssim \pi$ as the protocol in Ref. [27]. This protocol is described in detail in the Supplemental Material [26]. With this adaptive measurement strategy, we simulate clocks locked to between 1 and 4 ensembles for atom numbers from $N=4$ to 34 with an unlocked LO subject to both white and $1 / f$ noise with the constraint $\beta=0.3$. The stability of the clocks is plotted against the ensemble size $N$ in Fig. 3. For the adaptive protocol, we can apply the scheme of locking to several ensembles down to ensemble sizes of $N=4$ (7) for white $(1 / f)$ noise where we gain a factor of $\sim 2^{m-1}$ in $\sigma_{\gamma}^{2}(\tau)$ by locking the LO to $m$ ensembles. The minimal number of atoms is higher for $1 / f$ noise since the adaptive protocol is not as effective as for white noise where we have a better understanding of the a priori distribution in the Bayesian procedure [26]. It should be noted, however, that in principle it is only in the first ensemble that we need more atoms than for white noise since the feedback of the first ensemble whitens the noise. The adaptive protocol is thus more effective for the subsequent ensembles.

In conclusion, we have demonstrated a scheme for locking the LO in an atomic clock to $m$ ensembles of $N$ atoms each. For this scheme, the stability of the clock scales as $\sqrt{\gamma}\left(\gamma T_{1} N\right)^{-m / 2}$, where $T_{1}$ is the Ramsey time of the first ensemble. Our scheme thus provides an exponential improvement in the stability with the number of atoms. For the conventional Ramsey protocol, our scheme is applicable down to ensemble sizes of $N=20$ atoms while it is applicable down to ensemble sizes of $N=4$ (7) using an adaptive protocol. This makes the scheme relevant for atomic clocks with trapped ions. The performance of the protocol can be improved further by considering squeezed states, but this is beyond the scope of this article.

We gratefully acknowledge the support of the Lundbeck Foundation and the Danish National Research Foundation through QUANTOP. The research leading to these results has received funding from the European Research Council under the European Union's Seventh Framework Programme (FP/2007-2013)/ERC Grant Agreement No. 306576. We also thank D. Wineland, A. André, and M. Lukin for helpful discussions.

\footnotetext{
*borregaard@nbi.dk

†anders.sorensen@nbi.dk
}

[1] G. Santarelli, P. Laurent, P. Lemonde, A. Clairon, A. G. Mann, S. Chang, A. N. Luiten, and C. Salomon, Phys. Rev. Lett. 82, 4619 (1999).

[2] W. M. Itano, J. C. Bergquist, J. J. Bollinger, J. M. Gilligan, D. J. Heinzen, F. L. Moore, M. G. Raizen, and D. J. Wineland, Phys. Rev. A 47, 3554 (1993).

[3] D. J. Wineland, J. J. Bollinger, W. M. Itano, and D. J. Heinzen, Phys. Rev. A 50, 67 (1994).

[4] J. J. Bollinger, W. M. Itano, D. J. Wineland, and D. J. Heinzen, Phys. Rev. A 54, R4649 (1996).

[5] A. André, A. S. Sørensen, and M. D. Lukin, Phys. Rev. Lett. 92, 230801 (2004).

[6] V. Giovannetti, S. Lloyd, and L. Maccone, Nat. Photonics 5, 222 (2011).

[7] D. Leibfried, M. D. Barrett, T. Schaetz, J. Britton, J. Chiaverini, W. M. Itano, J. D. Jost, C. Langer, and D. J. Wineland, Science 304, 1476 (2004).

[8] I. D. Leroux, M. H. Schleier-Smith, and V. Vuletić, Phys. Rev. Lett. 104, 073602 (2010).

[9] J. Appel, P. J. Windpassinger, D. Oblak, U. B. Hoff, N. Kjærgaard, and E. S. Polzik, Proc. Natl. Acad. Sci. U.S.A. 106, 10960 (2009).

[10] A. Louchet-Chauvet, J. Appel, J. J. Renema, D. Oblak, N. Kjaergaard, and E. S. Polzik, New J. Phys. 12, 065032 (2010). 
[11] M. F. Riedel, P. Böhi, Y. Li, T. W. Hänsch, A. Sinatra, and P. Treutlein, Nature (London) 464, 1170 (2010).

[12] C. Gross, T. Zibold, E. Nicklas, J. Esteve, and M. K. Oberthaler, Nature (London) 464, 1165 (2010).

[13] S. A. Diddams, T. Udem, J.C. Bergquist, E. A. Curtis, R. E. Drullinger, L. Hollberg, W. M. Itano, W. D. Lee, C. W. Oates, K. R. Vogel, and D. J. Wineland, Science 293, 825 (2001).

[14] T. Udem, R. Holzwarth, and T.W. Hänsch, Nature (London) 416, 233 (2002).

[15] H. S. Margolis, H. S. Barwood, H. S. Huang, H. S. Klein, S. N. Lea, K. Szymaniec, and P. Gill, Science 306, 1355 (2004).

[16] M. Takamoto, F.-L. Hong, R. Higashi, and H. Katori, Nature (London) 435, 321 (2005).

[17] T. Rosenband, D. B. Hume, P. O. Schmidt, C. W. Chou, A. Brusch, L. Lorini, W. H. Oskay, R.E. Drullinger, T. M. Fortier, J.E. Stalnaker, S.A. Diddams, W.C. Swann, N. R. Newbury, W. M. Itano, D. J. Wineland, and J. C. Bergquist, Science 319, 1808 (2008).

[18] N. F. Ramsey, Molecular Beams (Oxford University Press, London, 1956).

[19] D. J. Berkeland, J. D. Miller, J. C. Bergquist, W. M. Itano, and D. J. Wineland, Phys. Rev. Lett. 80, 2089 (1998).
[20] D. J. Wineland, C. Monroe, W. M. Itano, D. Leibfried, B. E. King, and D. M. Meekhof, J. Res. Natl. Inst. Stand. Technol. 103, 259 (1998).

[21] Ph. Laurant, P. Lemonde, E. Simon, G. Santarelli, A. Clairon, N. Dimarcq, P. Petit, C. Audoin, and C. Salomon, Eur. Phys. J. D 3, 201 (1998).

[22] N. Shiga and M. Takeuchi, New J. Phys. 14, 023034 (2012).

[23] T. Rosenband and D. R. Leibrandt, arXiv:1303.6357.

[24] G. Dick, in Proceedings of the Nineteenth Annual Precise Time and Time Interval (PTTI) Applications and Planning Meeting, Redondo Beach, CA, 1987 (Naval Observatory, Washington, DC, 1987), p. 133.

[25] A. André, Ph.D. thesis, Harvard University, 2005.

[26] See Supplemental Material at http://link.aps.org/ supplemental/10.1103/PhysRevLett.111.090802 for details on our numerical simulations and the adaptive protocol.

[27] J. Borregaard and A.S. Sørensen, arXiv:1304.2288 [Phys. Rev. Lett. (to be published)].

[28] D. W. Berry and H. M. Wiseman, Phys. Rev. Lett. 85, 5098 (2000).

[29] B. L. Higgins, D. W. Berry, S. D. Bartlett, H. M. Wiseman, and G. J. Pryde, Nature (London) 450, 393 (2007). 